\title{
Gallbladder Perforation: Early Diagnosis Improves Survival
}

\author{
Bhupendra Kumar Jain • Pankaj Kumar Garg • \\ Debajyoti Mohanty
}

Received: 28 October 2014 / Accepted: 5 January 2015 /Published online: 23 January 2015

(C) Association of Surgeons of India 2015

\section{Dear Sir,}

We have read with interest the article "Diagnosis of gallbladder perforation - a puzzle!" by Cowksey SR et al. [1] While describing the clinical data of the 5 patients, the authors have not mentioned the physical findings and the clinical diagnosis in respect of these patients. Though the role of investigations like MR imaging, ERCP, cholescintigraphy, etc. has been mentioned by authors in reference to gallbladder perforation (GBP), none of their patients had undergone even ultrasonography. The physical findings and investigations are important in making preoperative diagnosis. None of the 5 patients was diagnosed preoperatively.

We have published our experience $[2,3]$ of managing 15 patients with GBP, 6 of whom could be diagnosed preoperatively as GBP. We would like to highlight some important aspect of this intriguing complication of cholecystitis, which presents as surgical emergency. The GBP presents to surgeon in emergency either as acute free perforation and generalized peritonitis (Niemeier type I) or subacute perforation, pericholecystic abscess, and localized peritonitis (Niemeier type II) [2]. The clinical course and prognosis of the two type of GBP is quite different. In our study, the type II perforation $(n=7)$ was associated with female preponderance ( $5 / 2$ vs. $3 / 5)$, high leucocute count $(22,600$ vs. 13,400$)$, high admissionoperation interval ( 93 vs. $17 \mathrm{~h}$ ), longer hospital stay (10.4 vs. 7.8 days), and high mortality (42.9 vs $12.5 \%)$.

B. K. Jain $(\bowtie) \cdot$ P. K. Garg

Department of Surgery, Guru Teg Bahadur Hospital and University College of Medical Sciences (University of Delhi), Dilshad Garden, Delhi 110095, India

e-mail: bhupendrakjain@gmail.com

D. Mohanty

Department of General Surgery, All India Institute of Medical

Sciences, Raipur, India
Preoperative diagnosis could be established in 3/7 patients by ultrasound and in other $4 / 4$ patients by contrast-enhanced computerized tomography in this group of patients.

The type I GBP presents as generalized peritonitis, and the patient is worked up for urgent laparotomy with clinical diagnosis of generalized peritonitis due to hollow viscus perforation. The diagnosis of GPB perforation is made on the operation table in this group of patients.

The type II perforation presents with peritoneal signs limited to epigastrium and right hypochondrium. The clinical diagnoses: acute pancreatitis, acute cholecystitis, empyema gallbladder, etc. are entertained in these patients, and they are managed initially with non-operative treatment. The delay in the operative treatment is responsible for development of uncontrolled sepsis, multi-organ failure, and high mortality in this group of patients.

High index of suspicion for GBP should be kept in patients with acute abdomen and features suggestive of acute pancreatitis or acute cholecystitis, particularly in those who have localized peritoneal signs, grossly elevated leukocyte counts, and normal serum amylase. These patients should be promptly investigated with ultrasound and CT scan if they do not respond rapidly to expectant line of treatment. Emergency cholecystectomy is the standard treatment.

Conflict of Interest All three authors (Bhupendra Kumar Jain, Pankaj Kumar Garg, and Debajyoti Mohanty) declare that they have no conflict of interest.

\section{References}

1. Chowksey SR, Baghel H, Sharma P, Singh B (2014) Diagnosis of gallbladder perforation - a puzzle! Ind J Surg 76:247-250

2. Jain BK, Prasad D, Mohanty D, Garg PK (2012) Gallbladder perforation: a great masquerader. Am Surg 78:E30-E32

3. Garg PK, Jain BK (2012) Gallbladder perforation: mystery continues to unravel. Int J Surg 10:272 